\title{
chaJOPEN
}

\section{Home blood pressure monitoring in the diagnosis and treatment of hypertension in pregnancy: a systematic review and meta-analysis}

\author{
Karen Tran MD MHSc, Raj Padwal MD MSc, Nadia Khan MD MSc, Mary-Doug Wright BSc MLS, \\ Wee Shian Chan MD MSc
}

Abstract

Background: Home blood pressure monitoring is increasingly used for pregnant individuals; however, there are no guidelines on such monitoring in this population. We assessed current practices in the prescription and use of home blood pressure monitoring in pregnancy.

Methods: We conducted a systematic review and meta-analysis of observational studies and randomized controlled trials (RCTs). We conducted a structured search through the MEDLINE (from 1946), Embase (from 1974) and CENTRAL (from 2018) databases up to Oct. 19, 2020. We included trials comparing office and home blood pressure monitoring in pregnant people. Outcomes included patient education, home blood pressure device, monitoring schedule, adherence, diagnostic thresholds for home blood pressure, and comparison between home and office measurements of blood pressure.

Results: We included in our review 21 articles on 19 individual studies (1 RCT, 18 observational) that assessed home and office blood pressure in pregnant individuals $(n=2843)$. We observed variation in practice patterns in terms of how home monitoring was prescribed. Eight (42\%) of the studies used validated home blood pressure devices. Across all studies, measurements were taken 3 to 36 times per week. Third-trimester home blood pressure corresponding to office blood pressure of 140/90 mm Hg after application of a conversion factor ranged from 118 to $143 \mathrm{~mm} \mathrm{Hg}$ (systolic) and from 76 to $92 \mathrm{~mm} \mathrm{Hg}$ (diastolic), depending on the patient population and methodology. Systolic and diastolic blood pressure values measured at home were lower than office values by 4 (95\% confidence interval [Cl] -6 to -3$) \mathrm{mm} \mathrm{Hg}$ and $3(95 \% \mathrm{Cl}-4$ to -2$) \mathrm{mm} \mathrm{Hg}$, respectively.

Interpretation: Many issues related to home blood pressure monitoring in pregnancy are currently unresolved, including technique, monitoring schedule and target values. Future studies should prioritize the use of validated home measuring devices and standardized measurement schedules and should establish treatment targets. PROSPERO registration: CRD42020147352

$\mathrm{H}$ ome blood pressure monitoring is routinely performed in adults for diagnosis and management of hypertension, ${ }^{1-5}$ because of its ease of use, frequency of measurements, cost effectiveness and availability. ${ }^{6}$ Home blood pressure correlates better with target endorgan damage and cardiovascular mortality than does office blood pressure. ${ }^{6}$ For these reasons, hypertension guidelines in Canada and internationally have strongly endorsed home blood pressure monitoring. ${ }^{1-5}$ Canadian obstetricians, family physicians, and obstetric internal medicine and maternalfetal medicine specialists frequently recommend home monitoring to their pregnant patients $s^{7,8}$ as a way to monitor for the development of hypertensive disorders of pregnancy. ${ }^{9}$ However, there is wide variation in how clinicians recommend home blood pressure monitoring in pregnancy, which highlights a lack of relevant studies. ${ }^{8}$

Despite the widespread use of home blood pressure monitoring in pregnancy, the best way to measure blood pressure at home, the thresholds at which to initiate antihypertensive medications and treatment targets are unclear. Extrapolating the experience of home monitoring from the general population is problematic because hemodynamic changes of pregnancy can lead to reductions in blood pressure at 18 to 24 weeks. Therefore, it is unclear whether "normal" blood pressure thresholds should vary according to different trimesters. Currently, Canadian guidelines in pregnancy state that

Competing interests: Raj Padwal is the CEO of mmHg Inc., provider of cloud-based digital health solutions. No other competing interests were declared.

This article has been peer reviewed.

Correspondence to: Karen Tran, karen.tran4 @ vch.ca

CMAJ Open 2021. DOI:10.9778/cmajo.20200099 
mean home blood pressure of $135 / 85 \mathrm{~mm} \mathrm{Hg}$ is equivalent to mean office blood pressure of 140/90 mm Hg, based on evidence from the nonpregnant population. ${ }^{10}$ This approach, however, has not been validated in the pregnant population. Recently, a meta-analysis based on individual patient data noted no difference between clinic and home blood pressure measurements in pregnancy; however, the study did not focus on how home blood pressure monitoring was prescribed. ${ }^{11}$

We conducted a systematic review and meta-analysis of observational studies and randomized controlled trials (RCTs) comparing home and office blood pressure measurements and evaluated how home blood pressure monitoring was prescribed in pregnancy.

\section{Methods}

\section{Study design}

We used the PICO (population, intervention, comparison, outcome[s]) framework to guide this systematic review and meta-analysis (Box 1). The study was registered with PROSPERO (CRD42020147352).

\section{Search strategy and study inclusion}

We conducted this systematic review in accordance with the Cochrane Handbook for Systematic Reviews of Interventions. ${ }^{15} \mathrm{We}$ conducted a structured search of the English-language literature in MEDLINE Ovid (1946 to Oct. 19, 2020), Embase Ovid (1974 to Oct. 19, 2020) and CENTRAL Ovid (2018 to Oct. 19, 2020). The complete search strategy is presented in Appendix 1 (available at www.cmajopen.ca/content/9/2/E642/ suppl/DC1).

We searched for observational studies and RCTs that compared home and office blood pressure measurements during the antepartum period. We excluded studies published in a language other than English, those published only as abstracts and those that did not compare home and office blood pressure measurements. The structured search was created by M.-D.W., a health research information specialist with systematic review experience.

We downloaded records from the database searches and imported them into an EndNote library to facilitate removal of duplicates and screening. Two of the authors (K.T., W.S.C.), working independently and in duplicate, assessed the records identified by the search strategy for inclusion, resolving any disagreements by discussion.

\section{Data extraction}

Two reviewers (K.T., W.S.C.) independently and in duplicate extracted data from full-text articles using a standardized data collection form. They resolved any disagreements through discussion.

We collected information about the brand of home blood pressure monitor, the home monitoring schedule, instructions to the patient, mean home blood pressure values and the upper limit of normal for home blood pressure readings, defined as the mean +2 standard deviations (SDs) or the 95 th percentile. We confirmed the validation of home blood pressure monitors
Box 1: Population, intervention, comparison and outcomes (PICO) framework

Population: The population of interest was pregnant individuals. Intervention: The intervention was antenatal use of home blood pressure monitoring, defined as use of a self-monitoring tool that allows patients to monitor their blood pressure in a setting other than the office of a health care provider.

Comparison: The eligible comparator was standard of care of blood pressure monitoring, defined as measurement of blood pressure in the office of a health care provider.

\section{Outcomes}

- Patient education: Presence of instructions provided to patients on how to adequately measure blood pressure at home, how information was communicated (i.e., written v. oral instructions) and thresholds to seek medical attention (i.e., safety mechanisms).

- Home blood pressure device: Type of home blood pressure monitor used and whether it is validated for use in the setting of pregnancy or preeclampsia (or both) according to the Association for the Advancement of Medical Instrumentation, ${ }^{12}$ the British Hypertension Society protocol ${ }^{13}$ or the international protocol for validation of automated blood pressure measuring devices. ${ }^{14}$

- Home blood pressure monitoring schedule: Frequency of measurement in 1 sitting, over 1 day and over 1 week, number of times in a day that blood pressure was measured, presence of discarded home readings and number of measurements recorded in 1 week.

- Adherence in performing home blood pressure measurements: Completion of the number of home blood pressure measurements as outlined in the research protocol.

- Diagnostic thresholds for home blood pressure measurements: Derivation of upper limit of home blood pressure readings for each trimester and method used to derive upper limit of normal home readings.

- Comparison between home and office blood pressure measurements: Mean home and office blood pressure for each trimester.

- Maternal pregnancy outcomes: Hypertensive disorders of pregnancy, preeclampsia.

- Fetal outcomes: Small at birth (birth weight < $1500 \mathrm{~g}$ ), small for gestational age $(<10$ th percentile for gestational age and sex), premature delivery $(<37 \mathrm{wk})$, perinatal death and admission to neonatal intensive care unit.

Inclusion criteria: Observational studies and parallel-group randomized controlled trials involving pregnant individuals and antenatal use of home and office blood pressure measurements.

Exclusion criteria: Studies that did not compare home and office blood pressure readings, abstracts and studies published in a language other than English.

for pregnancy using the STRIDE blood pressure website (www.stridebp.org). For office blood pressure measurements, we collected information about the method of measurement and the mean blood pressure in different trimesters.

In instances where we located multiple publications for the same study (e.g., substudy, extension), we used all available information to identify the relevant outcomes. To confirm unreported data, we reviewed study protocols and supplementary materials, and we contacted study authors to request any data missing from the available materials. 


\section{Quality measures and risk of bias}

For observational studies, we assessed study quality using the Newcastle-Ottawa Quality Assessment Scale for cohort studies. ${ }^{16}$ This scale is based on selection (4 items), comparability (1 item) and outcome (3 items) and generates a "star scoring system," which translates into good, fair or low quality. ${ }^{16}$ For RCTs, we assessed study quality using the Cochrane risk-ofbias tool for randomized trials. ${ }^{15}$ Two reviewers (K.T., W.S.C.), assessed study quality independently and resolved discrepancies by discussion.

\section{Statistical analysis}

For each study, we calculated the mean difference (as home minus office blood pressure measurements) and corresponding $95 \%$ confidence intervals (CIs). We assumed that the standard error of the mean difference calculated for each individual was independent of the standard errors for other individuals in the same study. We performed meta-analysis using a random-effects model. We assessed observed heterogeneity as the $I^{2}$ value, with values less than $25 \%$ denoting low heterogeneity. We created and visually inspected Egger funnel plots for asymmetry. We conducted a sensitivity analysis, including studies that used validated home blood pressure monitors in the pooled analysis.

We performed the analyses with Review Manager (RevMan) software, version 5.3 (Nordic Cochrane Centre, The Cochrane Collaboration, 2014). All $p$ values are 2-tailed.

\section{Ethics approval}

Our systematic review and meta-analysis used publicly available aggregate data; as such, ethics approval was not required.

\section{Results}

Our search strategy yielded 1366 unique citations, of which 58 were eligible for full-text review. We included 21 articles from 19 individual studies in our systematic review (18 observational studies and 1 RCT) (Figure 1, Table 1). Three of the articles involved the same cohort of patients, from the Babies' and Their Parents' Longitudinal Observation in Suzuki Memorial Hospital on Intrauterine Period (BOSHI) study. ${ }^{17-19}$

We assessed the 19 studies ( $n=2843$ pregnant participants) in our analysis. The studies, published from 1987 to 2020 , included pregnant patients who were normotensive $(n=7$ studies), as well as participants who had chronic hypertension $(n=7)$, hypertensive disorders of pregnancy $(n=4)$, high risk of preeclampsia $(n=1)$, white coat hypertension $(n=3)$ or diabetes $(n=1)$. Study characteristics are summarized in Table 1 .

\section{Quality of evidence}

Using the Newcastle-Ottawa Scale for quality assessment, we judged most studies to be of fair ${ }^{23-31,33}$ or $\operatorname{good}^{17-19,32,34,35,37}$ quality. Only 3 studies were deemed to be of poor quality ${ }^{20-22}$ with high risk of bias. Using the Cochrane risk-of-bias tool, we judged the RCT to be at low risk of bias. ${ }^{36}$ Although the participants, clinicians and investigators were not blinded, standardized methods for office measurements of blood pressure were completed to minimize bias.

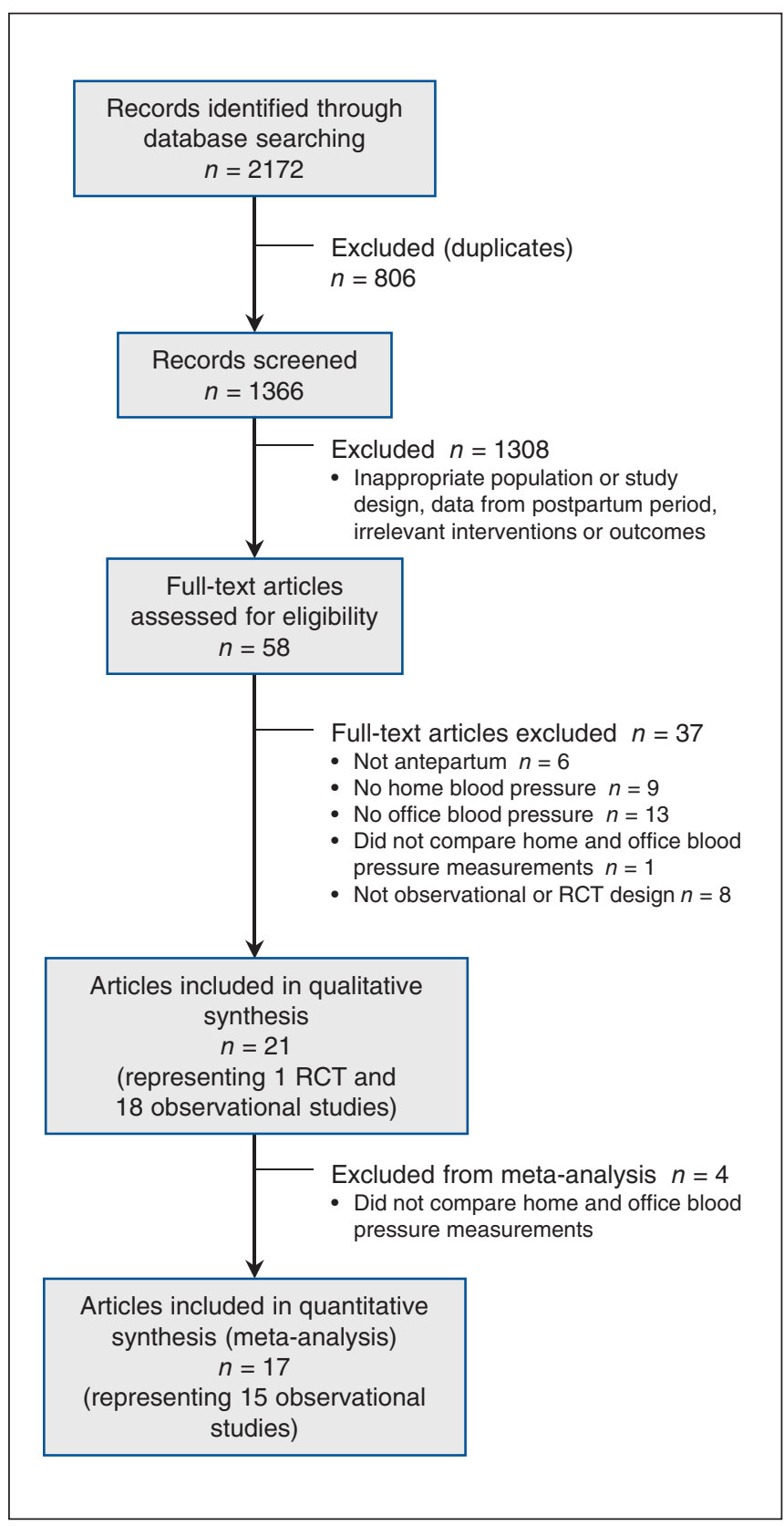

Figure 1: PRISMA flow diagram. Note: RCT = randomized controlled trial.

\section{Synthesis of results}

\section{Patient education and monitoring}

Eighteen of the 19 studies provided either verbal or written instructions to participants on how to monitor blood pressure at home. Five studies used telemonitoring ${ }^{20,22,30}$ or medical apps ${ }^{32,36}$ to transmit measurements to clinicians. Five studies provided instructions to patients on seeking medical advice for their hypertension. ${ }^{27,29,31,32,36}$ These triggering blood pressure values were variable: $\geq 140 / 90 \mathrm{~mm} \mathrm{Hg}$ with proteinuria or $\geq 160 / 100 \mathrm{~mm} \mathrm{Hg}$ without proteinuria; ${ }^{27}$ systolic blood pressure $\geq 155 \mathrm{~mm} \mathrm{Hg}$ or diastolic 


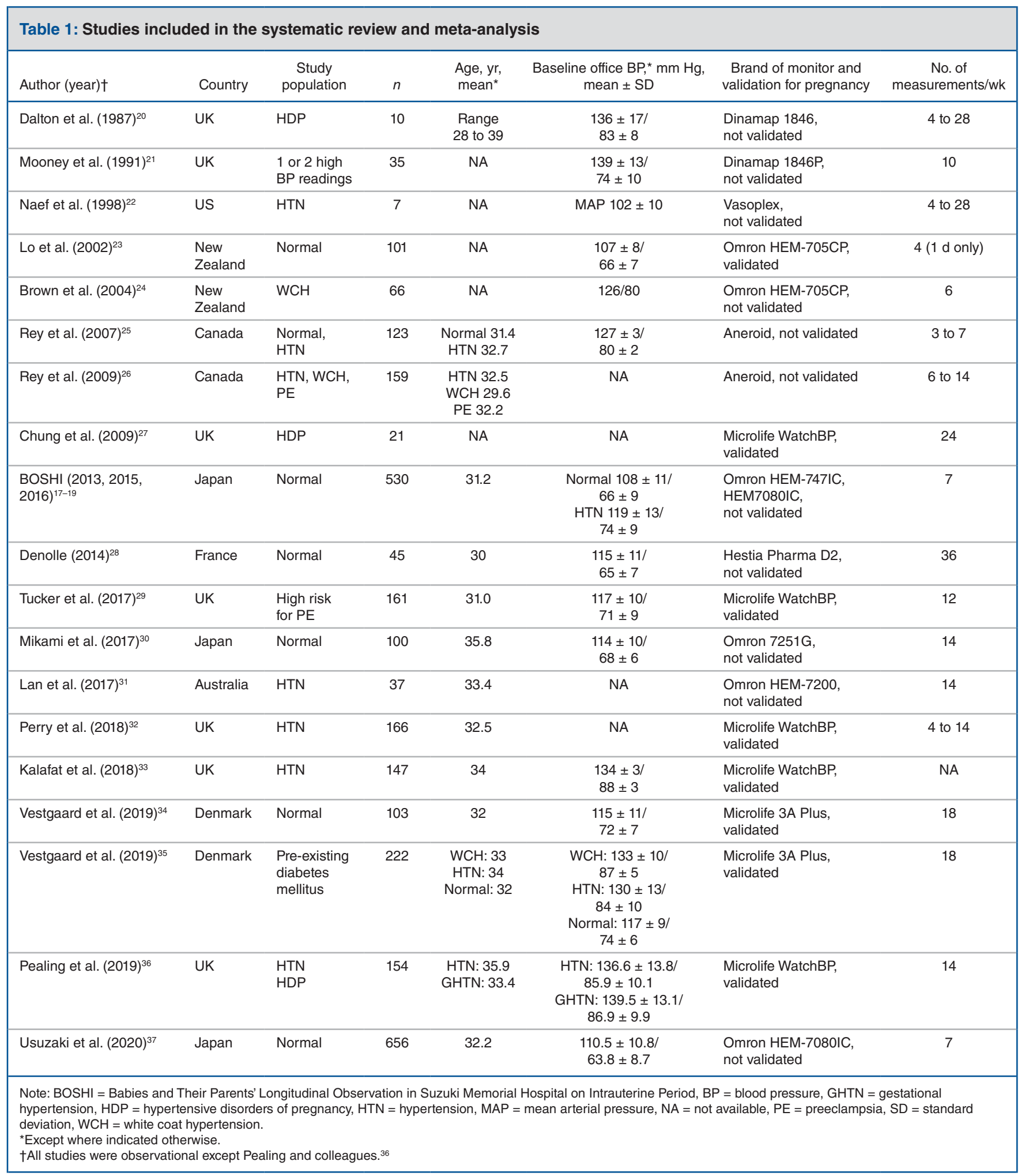

blood pressure $\geq 100 \mathrm{~mm} \mathrm{Hg} ;{ }^{32} \geq 140 / 90 \mathrm{~mm} \mathrm{Hg}$ or $\leq 100 / 60 \mathrm{~mm} \mathrm{Hg}^{31}$ and systolic blood pressure $\geq 150 \mathrm{~mm} \mathrm{Hg}$ or diastolic blood pressure $\geq 100 \mathrm{~mm} \mathrm{Hg.}{ }^{29,36}$ Two studies implemented a colour-coded pamphlet with detailed instructions and follow-up..$^{29,36}$

\section{Device selection and validation}

Eight (42\%) of the 19 studies used a validated home blood pressure monitor approved for use in pregnancy (Watch BP, Microlife; 3A Plus, Microlife; HEM-705CP, Omron), ${ }^{27,29,31-36}$ with the first of these being the most common (5 studies [26\%]). 


\section{Frequency and timing of home blood pressure measurement}

Blood pressure was measured at home between 1 and 6 times per day. Measuring blood pressure in the morning and evening for 1 week was the most common method described (5 studies [26\%]). The total number of measurements recorded per week ranged from 3 to 36 . One study discarded the first day's readings, and then averaged the remaining results. ${ }^{31}$

\section{Adherence in performance of home monitoring}

Adherence to home blood pressure monitoring was reported in 4 studies. ${ }^{19,27,31,36}$ Three of the studies reported high adherence (76\% to $92 \%),{ }^{27,31,36}$ but Iwama and colleagues ${ }^{19}$ noted that only $9.4 \%$ of participants in their study measured blood pressure daily. Pealing and colleagues ${ }^{36}$ found that women with gestational or chronic hypertension measured their blood pressure on a median of 6.1 and 5.5 days per week, respectively.

\section{Diagnostic thresholds for home blood pressure measurements}

Proposed diagnostic thresholds for blood pressure monitored at home varied by patient population, methodology and trimester (Table 2). Five studies calculated the diagnostic thresholds by applying to the home blood pressure data a relative metric derived from the office data for the same sample..$^{23,25,28,30,34}$ Depending on the method used, the diagnostic threshold for home blood pressure for the third trimester was 118/76 mm Hg (95 th percentile cut-off); ${ }^{28}$ 143/92 $\mathrm{mm} \mathrm{Hg}$ (90th percentile cutoff); ${ }^{25} 121 / 80 \mathrm{~mm} \mathrm{Hg} ;{ }^{28} 123 / 78 \mathrm{~mm} \mathrm{Hg}^{34}$ and 138/88 $\mathrm{mm} \mathrm{Hg}^{23}$ (all with 2 SD cut-off); and 136/89 mm Hg (cut-off based on regression line from standardized major axis methods). ${ }^{30}$

Home blood pressure thresholds were lower for the first and second trimesters than for the third trimester. Further- more, studies involving participants with normal blood pressure $^{28,34}$ had lower thresholds than studies with participants who had chronic hypertension or who developed hypertensive disorders of pregnancy. ${ }^{23,25,30}$

\section{Comparison between home and office measurements}

Meta-analysis of aggregate data for 2495 pregnant patients (15 observational studies) showed that systolic blood pressure measured at home was lower than that measured in the office by $4(95 \% \mathrm{CI}-6$ to -3$) \mathrm{mm} \mathrm{Hg}$, with $I^{2}$ of $79 \%$. Diastolic blood pressure measured at home was lower than that measured in the office by 3 (95\% CI -4 to -2$) \mathrm{mm} \mathrm{Hg}$, with $I^{2}$ of $82 \%$ (Figure 2).

Sensitivity analysis showed that the mean difference between home and office measurements, based on validated home monitors, was -4 (95\% CI -7 to -2$) \mathrm{mm} \mathrm{Hg}$ for systolic blood pressure and -4 (95\% CI -7 to -2$) \mathrm{mm} \mathrm{Hg}$ for diastolic blood pressure (Appendix 2, available at www.cmajopen.ca/ content/9/2/E642/suppl/DC1). Significant heterogeneity was observed ( $I^{2} 80 \%$ to $88 \%$ ). Furthermore, asymmetry was noted in the Egger funnel plots, which suggested publication bias (Appendix 3, available at www.cmajopen.ca/content/9/2/ E642/suppl/DC1).

\section{Relation of home blood pressure measurements to pregnancy outcomes}

Two of the studies reported pregnancy outcomes. Perry and colleagues $^{32}$ noted no difference in maternal or fetal outcomes between home blood pressure monitoring and usual care in the maternity day unit; however, their study was not powered to detect such differences. Home blood pressure monitoring was associated with a reduction in hypertension-related visits (6.5 visits v. 8 visits, $p=0.003) .{ }^{32}$ In contrast, in another study ${ }^{36}$ women with chronic hypertension randomly assigned

Table 2: Summary of proposed definitions for trimester-specific upper limit of normal home blood pressure

\begin{tabular}{|c|c|c|c|c|c|c|c|c|}
\hline Reference & Population & Method & $\begin{array}{l}\text { SBP, } \\
\mathrm{mm} \mathrm{Hg}\end{array}$ & $\begin{array}{l}\text { DBP, } \\
\mathrm{mm} \mathrm{Hg}\end{array}$ & $\begin{array}{l}\text { SBP, } \\
\mathrm{mm} \mathrm{Hg}\end{array}$ & $\begin{array}{l}\text { DBP, } \\
\mathrm{mm} \mathrm{Hg}\end{array}$ & $\begin{array}{l}\text { SBP, } \\
\mathrm{mm} \mathrm{Hg}\end{array}$ & $\begin{array}{l}\text { DBP, } \\
\mathrm{mm} \mathrm{Hg}\end{array}$ \\
\hline Lo et al. ${ }^{23}$ & Normal & Mean \pm 2 SD & 132 & 82 & 130 & 79 & $133 / 138^{*}$ & $81 / 88^{*}$ \\
\hline Rey et al. ${ }^{25}$ & $\begin{array}{l}\text { Normal and } \\
\text { hypertensive }\end{array}$ & 90th percentile & $139 \dagger$ & $89 \dagger$ & 137 & 87 & $138 / 140 / 143 \ddagger$ & 89/90/92‡ \\
\hline Denolle $^{28}$ & Normal & 95th percentile & 116 & 70 & 113 & 70 & 118 & 76 \\
\hline Mikami et al. ${ }^{30}$ & Normal & $\begin{array}{l}\text { Regression line from } \\
\text { standardized major } \\
\text { axis methods }\end{array}$ & 120.8 & 83.5 & 124/127§ & 84/86§ & 136 & 89 \\
\hline $\begin{array}{l}\text { Vestgaard et } \\
\text { al. }^{34}\end{array}$ & Normal & Mean \pm 2 SD & 117 & 74 & 116 & 73 & 123 & 78 \\
\hline
\end{tabular}


A)

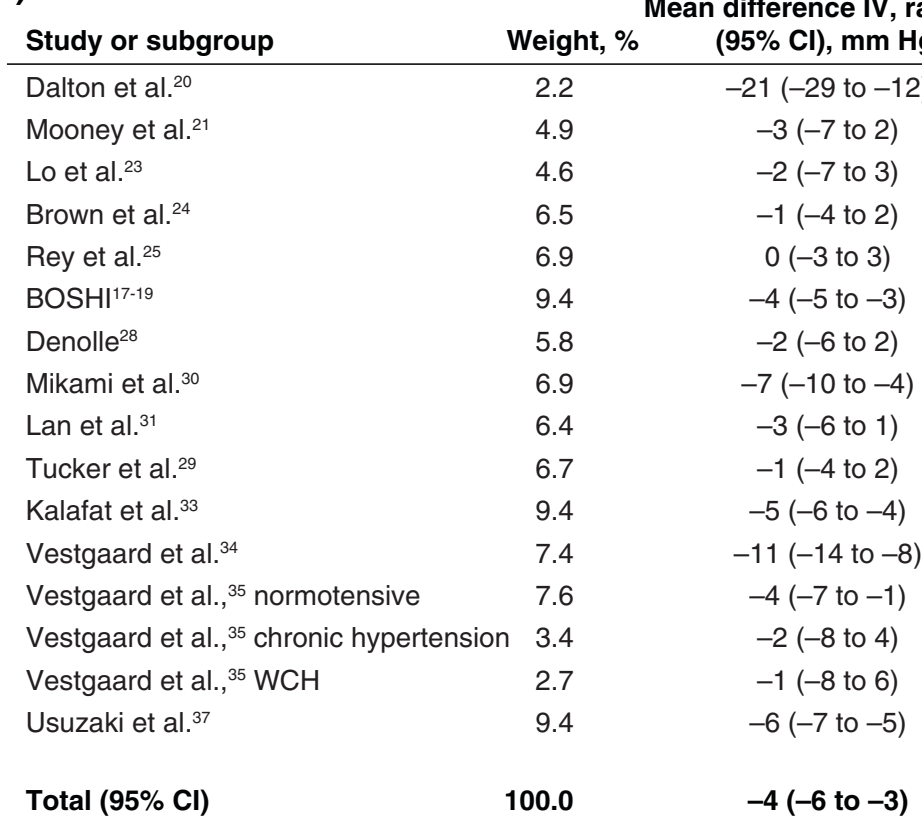

Heterogeneity: $\tau^{2}=5.16 ; \chi^{2}=72.49, \mathrm{df}=15(p<0.001) ; l^{2}=79 \%$

Test for overall effect: $Z=5.85(p<0.001)$

\begin{tabular}{|c|c|c|c|c|}
\hline-20 & -10 & 0 & 10 & 20 \\
\hline
\end{tabular}

B)

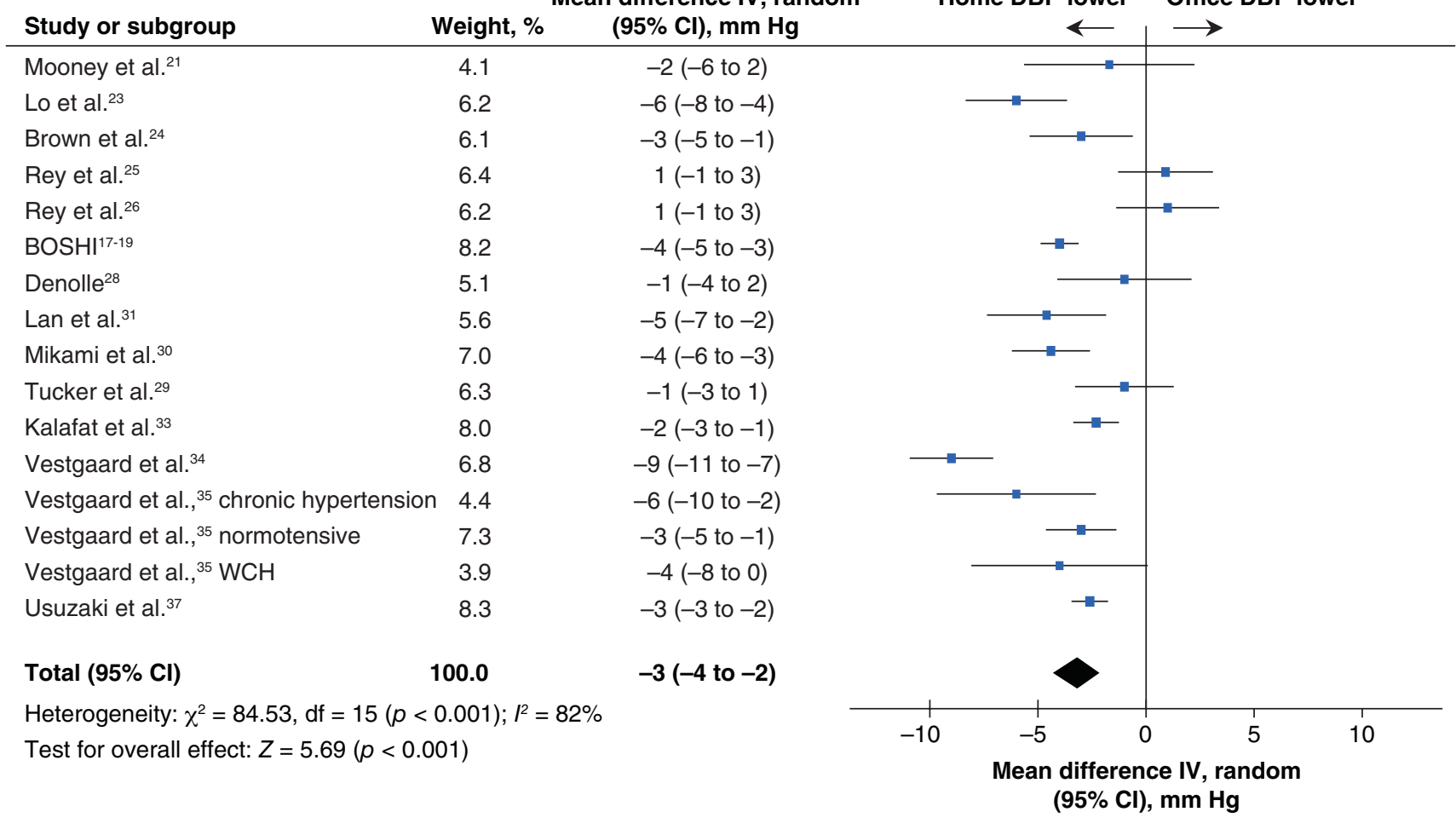

Figure 2: Forest plots for comparison of differences in mean systolic blood pressure (SBP; panel A) and diastolic blood pressure (DBP; panel B) between home and office blood pressure measurements in pregnancy, using random effects. The mean difference was calculated as home BP - office BP. Note: $\mathrm{Cl}=$ confidence interval, IV = inverse variance, $\mathrm{WCH}=$ white coat hypertension. 
to self-monitoring of blood pressure had increased odds of preeclampsia compared with routine care (odds ratio [OR] $2.9,95 \%$ CI 0.9 to 9.1 ); however, that study was not powered to detect differences in secondary outcomes.

Postintervention surveys showed that home blood pressure monitoring was easy to use. ${ }^{22,38}$ Home measurements changed the management plans for $10 \%$ of patients in one of the studies, but almost half of the patients did not follow instructions properly. ${ }^{27}$

\section{Interpretation}

Despite increased adoption of home blood pressure monitoring in the management of pregnant individuals with hypertension, deriving an evidence-based approach from the current literature is difficult. In our aggregate data meta-analysis, we found that mean home blood pressure was lower than mean office blood pressure in pregnant individuals by a small amount (4 mm Hg for systolic blood pressure and $3 \mathrm{~mm} \mathrm{Hg}$ for diastolic blood pressure), but with substantial variation in this difference across studies. Overall, the study quality was judged to be moderate, as the majority of studies were of fair to good quality. However, problems lie in the execution of home blood pressure monitoring, as most studies did not report individual-level differences between home and office blood pressure and most did not use validated devices.

Home blood pressure devices interpret an oscillometric signal, which is reduced in pregnancy because of changes in vascular compliance, intravascular volume and vascular wall edema; hence, these devices may underestimate blood pressure. ${ }^{32,34}$ With the use of nonvalidated blood pressure devices, hypertensive disorders of pregnancy may be underdiagnosed and undertreated by $48 \%$ and $80 \%$, respectively. ${ }^{39}$ Furthermore, in a real-world setting, few pregnant patients use a validated home blood pressure monitor specific for pregnancy. ${ }^{40}$ This may stem from the limited availability of home monitors that have been validated in pregnancy, ${ }^{41}$ costs and clinicians' lack of knowledge about validated home monitors for pregnancy. STRIDE BP (www.stridebp.org) is an international nonprofit organization that summarizes validated home blood pressure monitors for clinicians.

The method used to determine home blood pressure varied among studies. Between 3 and 36 readings per week were used to calculate participants' average home blood pressure, and no studies derived prognostic outcome algorithms based on these readings. In contrast to the nonpregnant population who were not hospitalized, with reported adherence of $59.7 \%$ (range $52.1 \%$ to $67.3 \%$ ), ${ }^{42}$ adherence with home blood pressure monitoring among pregnant people ranged between $76 \%$ and $96 \%$. Even when clear instructions were provided to patients, they were followed correctly only half the time. ${ }^{27}$

To have accurate measurement of blood pressure at home, patient education is vital. For the nonpregnant population, a minimum of 12 readings is needed for home blood pressure monitoring to be valid; ${ }^{6}$ more recently, 3 days of blood pressure readings appeared sufficient to predict home blood pressure readings. ${ }^{43}$ A pragmatic monitoring schedule for pregnancy is needed to balance the validity of home blood pressure readings with patient adherence.

We observed that the proposed diagnostic thresholds for hypertension in the third trimester of pregnancy varied across studies, ranging from 118 to $143 \mathrm{~mm} \mathrm{Hg}$ for systolic blood pressure and from 76 to $92 \mathrm{~mm} \mathrm{Hg}$ for diastolic blood pressure, because of heterogeneity and methodology. ${ }^{23,25,28,30,34}$ Instead of deriving diagnostic thresholds using relative metrics based on office data, it would be preferable to collect high-quality prognostic data to determine home blood pressure thresholds at which the risk of clinically important complications begins to rise.

Our meta-analysis showed that home measurements of systolic and diastolic blood pressure were lower than office measurements by 4 (95\% CI -6 to -3$) \mathrm{mm} \mathrm{Hg}$ and 3 (95\% CI -4 to -2$) \mathrm{mm} \mathrm{Hg}$, respectively. These results differ from those reported by Tucker and colleagues ${ }^{11}$ in their meta-analysis based on individual patient data, which showed no differences between home and office blood pressure measurements (mean difference of 1.5 to $2.2 \mathrm{~mm} \mathrm{Hg}$ for systolic blood pressure and 0.7 to $1.5 \mathrm{~mm} \mathrm{Hg}$ for diastolic blood pressure). Both metaanalyses showed significant heterogeneity in the data $\left(I^{2}>\right.$ $80 \%$, which suggests substantial variability in study design, population, measurement devices and method of measurement. Given that the literature to date is not definitive, clinical implementation of procedures for patients and assumptions about the equivalency of home and office blood pressure are premature, and more research and evaluation are needed.

In their meta-analysis, Kalafat and colleagues ${ }^{44}$ noted that home monitoring of blood pressure reduced the risk of induction of labour (OR $0.55,95 \%$ CI 0.36 to 0.88 ), prenatal hospital admissions (OR $0.31,95 \%$ CI 0.19 to 0.49 ) and diagnosis of preeclampsia (OR $0.50,95 \%$ CI 0.31 to 0.81 ) and also reduced the number of antenatal visits (standard mean difference $-0.49,95 \%$ CI -0.82 to -0.16$)$. However, strong clinical heterogeneity, low quality of evidence and small sample size were important limitations in that study. ${ }^{44}$

Home blood pressure monitoring is an important method when managing the care of individuals with hypertensive disorders of pregnancy, as it can detect rapid and acute blood pressure changes. Qualitative analysis has shown that patients perceive clinical benefit with home blood pressure measurement, including decreased need for antenatal visits, ${ }^{30}$ timely adjustments of antihypertensive medications ${ }^{28}$ and ease of use. ${ }^{23}$

Home blood pressure monitoring has the potential to revolutionize the care of pregnant patients with hypertension. However, on the basis of current studies, its implementation is uncertain. Future studies evaluating home blood pressure monitors for hypertensive disorders of pregnancy should use validated devices, collect reference data from groups of healthy pregnant patients, use guideline-concordant schedules for home monitoring, define diagnostic and treatment thresholds based on home blood pressure measurements, and collect outcome data.

\section{Limitations}

This study had several limitations. Limiting the search strategy to English publications and omitting grey literature from 
the review might have contributed to publication bias. We hypothesized that studies showing no difference between home and office blood pressure might not have been published. Moreover, excluding published abstracts in the search strategy may have contributed to publication bias. We hypothesize that published abstracts are more likely to show differences between home and office blood pressure readings, which may be exaggerated as the data may be preliminary and not peer reviewed.

There is a lack of contemporary RCTs on this topic (with only 1 study identified in our search) and also a lack of outcome data for home blood pressure monitoring in pregnancy more generally. These limitations highlight the need for highquality RCTs to assess this form of care in pregnant patients.

The quality of available data was poor, because of low rigour in how home blood pressure monitoring was conducted, including lack of validated home blood pressure devices, lack of patient education, lack of standardization of home monitoring schedules and lack of reporting of withinwomen differences, all of which may influence the interpretation of our meta-analysis.

The measure of homogeneity, $I^{2}(>80 \%)$, was likely an underestimate because of the assumption of independence of home and office measurements, which highlights the relatively high heterogeneity in our meta-analysis. Future studies with strong emphasis on the rigorous use of home blood pressure monitoring in pregnancy are needed.

\section{Conclusion}

This systematic review and meta-analysis showed that current studies do not provide adequate guidance with respect to the use of home blood pressure monitoring by pregnant patients. More studies are needed, and these should focus on defining diagnostic and treatment thresholds for home blood pressure and clarifying the relation between home monitoring and clinically important outcomes.

\section{References}

1. Nerenberg KA, Zarnke KB, Leung AA, et al.; Hypertension Canada. Hypertension Canada's 2018 guidelines for diagnosis, risk assessment, prevention, and treatment of hypertension in adults and children. Can 7 Cardiol 2018;34:506-25.

2. Imai Y, Kario K, Shimada K, et al.; Japanese Society of Hypertension, Committee for Guidelines for Self-monitoring of Blood Pressure at Home. The Japanese Society of Hypertension guidelines for self-monitoring of blood pressure at home (second edition). Hypertens Res 2012;35:777-95.

3. Krause T, Lovibond K, Caulfield M, et al.; Guideline Development Group. Management of hypertension: summary of NICE guidance. BMF 2011;343:d4891.

4. Williams B, Mancia G, Spiering W, et al.; Authors/Task Force Members. 2018 ESC/ESH guidelines for the management of arterial hypertension: the task force for the management of arterial hypertension of the European Society of Cardiology and the European Society of Hypertension. 7 Hypertens 2018;36:1953-2041.

5. Whelton PK, Carey RM, Aronow WS, et al. 2017 ACC/AHA/AAPA/ABC/ ACPM/AGS/APhA/ASH/ASPC/NMA/PCNA guideline for the prevention, detection, evaluation, and management of high blood pressure in adults: a report of the American College of Cardiology/American Heart Association Task Force on Clinical Practice Guidelines. Circulation 2018;138:e484-594.

6. Parati G, Stergiou GS, Asmar R, et al.; ESH Working Group on Blood Pressure Monitoring. European Society of Hypertension guidelines for blood pressure monitoring at home: a summary report of the Second International Consensus Conference on Home Blood Pressure Monitoring. 7 Hypertens 2008;26:1505-26.

7. Dehaeck U, Thurston J, Gibson P, et al. Blood pressure measurement for hypertension in pregnancy. 7 Obstet Gynaecol Can 2010;32:328-34.
8. Tran KC, Potts J, Robertson J, et al. Out-of-office blood pressure measurement for the diagnosis of hypertension in pregnancy: survey of Canadian obstetric medicine and maternal fetal medicine specialists. Obstet Med 2020;13:20-4.

9. Magee LA, von Dadelszen P, Chan S, et al.; Chips Pilot Trial Collaborative Group. Women's views of their experiences in the CHIPS (Control of Hypertension in Pregnancy Study) pilot trial. Hypertens Pregnancy 2007;26:371-87.

10. Magee LA, Pels A, Helewa M, et al.; Canadian Hypertensive Disorders of Pregnancy (HDP) Working Group. Diagnosis, evaluation, and management of the hypertensive disorders of pregnancy. Pregnancy Hypertens 2014;4:105-45.

11. Tucker KL, Bankhead C, Hodgkinson J, et al. How do home and clinic blood pressure readings compare in pregnancy? Hypertension 2018;72:686-94.

12. ANSI/AAMI/ISO 81060-2:2013 non-invasive sphygmomanometers - part 2: Clinical investigation of automated measurement type. Arlington (VA): Association for the Advancement of Medical Instrumentation; 2013. Available: www. aami.org (accessed 2018 May 1).

13. O'Brien E, Petrie J, Littler W, et al. The British Hypertension Society protocol for the evaluation of blood pressure measuring devices. 7 Hypertens 1993;11 Suppl 2:S43-S62.

14. O'Brien E, Atkins N, Stergiou G, et al. European Society of Hypertension international protocol revision 2010 for the validation of blood pressure measuring devices in adults. Blood Press Monit. 2010;15:23-38.

15. Higgins JPT, Green S, editors. Cochrane handbook for systematic reviews of interventions. Version 5.1.0. Oxford (UK): Cochrane Collaboration; 2011.

16. Wells GA, Shea B, O'Connell D, et al. The Newcastle-Ottawa Scale (NOS) for assessing the quality of nonrandomized studies in meta-analyses. Ottawa: Ottawa Hospital Research Institute. Available: www.ohri.ca/programs/clinical_ epidemiology/oxford.asp (accessed 2019 May 15).

17. Ishikuro M, Obara T, Metoki H, et al. Blood pressure measured in the clinic and at home during pregnancy among nulliparous and multiparous women: the BOSHI study. Am 7 Hypertens 2013;26:141-8.

18. Ishikuro M, Obara T, Metoki H, et al. Differences between clinic and home blood pressure measurements during pregnancy. F Hypertens 2015;33:1492-3.

19. Iwama N, Metoki H, Ohkubo T, et al.; BOSHI Study Group. Maternal clinic and home blood pressure measurements during pregnancy and infant birth weight: the BOSHI study. Hypertens Res 2016;39:151-7.

20. Dalton KJ, Manning K, Robarts PJ, et al. Computerized home telemetry of maternal blood pressure in hypertensive pregnancy. Int 7 Biomed Comput 1987;21:175-87.

21. Mooney P, Jaspar A, Cartwright $W$, et al. No difference between home and clinic blood pressure measurements in pregnancy: a computerized telemetric study. 7 Perinat Med 1991;19:133-9.

22. Naef RW 3rd, Perry KG Jr, Magann EF, et al. Home blood pressure monitoring for pregnant patients with hypertension. F Perinatol 1998;18:226-9.

23. Lo C, Taylor RS, Gamble G, et al. Use of automated home blood pressure monitoring in pregnancy: Is it safe? Am $\mathcal{7}$ Obstet Gynecol 2002;187:1321-8.

24. Brown MA, McHugh L, Mangos G, et al. Automated self-initiated blood pressure or 24-hour ambulatory blood pressure monitoring in pregnancy? B7OG 2004;111:38-41.

25. Rey E, Pilon F, Boudreault J. Home blood pressure levels in pregnant women with chronic hypertension. Hypertens Pregnancy 2007;26:403-14

26. Rey E, Morin F, Boudreault J, et al. Blood pressure assessments in different subtypes of hypertensive pregnant women: office versus home patient- or nurse-measured blood pressure. Hypertens Pregnancy 2009;28:168-77.

27. Chung Y, de Greeff A, Shennan A. Validation and compliance of a home monitoring device in pregnancy: Microlife WatchBP Home. Hypertens Pregnancy 2009;28:348-59.

28. Denolle T. [Home blood pressure measurement and pregnancy]. Presse Med 2014;43:827-30. Article in French.

29. Tucker KL, Taylor KS, Crawford C, et al. Blood pressure self-monitoring in pregnancy: examining feasibility in a prospective cohort study. BMC Pregnancy Childbirth 2017;17:442.

30. Mikami Y, Takai Y, Era S, et al. Provisional criteria for the diagnosis of hypertension in pregnancy using home blood pressure measurements. Hypertens Res 2017;40:679-84.

31. Lan PG, Hyett J, Gillin AG. Home blood pressure measurement in women with pregnancy-related hypertensive disorders. Pregnancy Hypertens 2017;10:213-9.

32. Perry H, Sheehan E, Thilaganathan B, et al. Home blood-pressure monitoring in a hypertensive pregnant population. Ultrasound Obstet Gynecol 2018;51:524-30.

33. Kalafat E, Mir I, Perry H, et al. Is home blood pressure monitoring in hypertensive disorders of pregnancy consistent with clinic recordings? Ultrasound Obstet Gynecol 2018;52:515-21.

34. Vestgaard M, Carstens Søholm J, Kjærhus Nørgaard S, et al. Home blood pressure in pregnancy - the upper reference limit. Blood Press Monit 2019;24:191-8.

35. Vestgaard M, Ásbjörnsdóttir B, Ringholm L, et al. White coat hypertension in early pregnancy in women with pre-existing diabetes: prevalence and pregnancy outcomes. Diabetologia 2019;62:2188-99.

36. Pealing LM, Tucker KL, Mackillop LH, et al.; OPTIMUM-BP Investigators. A randomised controlled trial of blood pressure self-monitoring in the management of hypertensive pregnancy. OPTIMUM-BP: a feasibility trial. Pregnancy Hypertens 2019;18:141-9.

37. Usuzaki T, Ishikuro $\mathrm{M}$, Metoki $\mathrm{H}$, et al. Comparison among research, home, and office blood pressure measurements for pregnant women: the 
TMM BirThree Cohort Study. 7 Clin Hypertens (Greenwich) 2020;22: 2004-13.

38. Hinton L, Tucker KL, Greenfield SM, et al. Blood pressure self-monitoring in pregnancy (BuMP) feasibility study; a qualitative analysis of women's experiences of self-monitoring. BMC Pregnancy Childbirth 2017;17:427.

39. Nzelu D, Yeung F, Couderq D, et al. An inaccurate automated device negatively impacts the diagnosis and treatment of gestational hypertension. Pregnancy Hypertens 2017;10:28-33.

40. Tremonti C, Beddoe J, Brown MA. Reliability of home blood pressure monitoring devices in pregnancy. Pregnancy Hypertens 2017;8:9-14.

41. Bello NA, Woolley JJ, Cleary KL, et al. Accuracy of blood pressure measurement devices in pregnancy: a systematic review of validation studies. Hypertension 2018;71:326-35.

42. Celler B, Argha A, Varnfield M, et al. Patient adherence to scheduled vital sign measurements during home telemonitoring: analysis of the intervention arm in a before and after trial. 7MIR Med Inform 2018;6:e15.

43. Hodgkinson JA, Stevens R, Grant S, et al. Schedules for self-monitoring blood pressure: a systematic review. Am 7 Hypertens 2019;32:350-64.

44. Kalafat E, Benlioglu C, Thilaganathan B, et al. Home blood pressure monitoring in the antenatal and postpartum period: a systematic review metaanalysis. Pregnancy Hypertens 2020;19:44-51.

Affiliations: Division of General Internal Medicine (Tran, Khan, Chan), Department of Medicine, University of British Columbia; Centre for Health Evaluation and Outcome Sciences (Tran, Khan); and Apex Information (Wright), Vancouver, BC; Division of General Internal Medicine (Padwal), Department of Medicine, University of Alberta, Edmonton, Alta.
Contributors: Karen Tran and Wee Shian Chan led the overall project. Karen Tran and Wee Shian Chan conceived the project. Karen Tran and Mary-Doug Wright designed the search strategy. Karen Tran and Wee Shian Chan performed the review. Karen Tran, Raj Padwal, Nadia Khan and Wee Shian Chan analyzed and interpreted the data. Karen Tran drafted the manuscript, and Raj Padwal, Nadia Khan, Mary-Doug Wright and Wee Shian Chan critically revised it for important intellectual content. All of the authors approved the version to be published and agreed to be accountable for all aspects of the work.

Funding: No funding was received for this study.

Content licence: This is an Open Access article distributed in accordance with the terms of the Creative Commons Attribution (CC BY-NC-ND 4.0) licence, which permits use, distribution and reproduction in any medium, provided that the original publication is properly cited, the use is noncommercial (i.e., research or educational use), and no modifications or adaptations are made. See: https://creativecommons.org/licenses/by-nc-nd/4.0/

Data sharing: All extracted data are available for sharing. Others may access the data by contacting the corresponding author.

Acknowledgement: The authors acknowledge Dr. Amélie Boutin for her contributions to the statistical analysis.

Supplemental information: For reviewer comments and the original submission of this manuscript, please see www.cmajopen.ca/content/9/2/ E642/suppl/DC1. 